\title{
Survey to assess management of abdominal pain and cramping in South African pharmacy chains
}

\author{
D Webb' ${ }^{1}$ L Jagwanth ${ }^{2}$ \\ ${ }^{1}$ Pattacus Medical Writing, South Africa \\ ${ }^{2}$ Sanofi Consumer Healthcare, South Africa \\ Corresponding author, email: dawebb@mweb.co.za
}

\begin{abstract}
Background: Most consumers with abdominal cramps and pain choose to treat them with over-the-counter (OTC) medication. Currently there are no data evaluating the approach of South African pharmacy staff to abdominal cramps and pain. The purpose of this survey was to evaluate the approach of pharmacy healthcare providers (HCPs) to abdominal pain and cramping and attitudes towards Buscopan (hyoscine butyl bromide) for treatment of abdominal cramps.
\end{abstract}

Method: An online and face-to-face survey was conducted with 142 pharmacists and 82 pharmacist's assistants from two major retail pharmacy chains. The study was commissioned by an independent research company.

Results: Pharmacists and pharmacist's assistants reported providing advice to an average of 13 patients with abdominal cramps weekly. The majority of HCPs recommended treatment based on symptoms, potential for drug interactions and safety. Only approximately half of consumers always followed the advice of the HCP. HCPs commonly saw patients who had taken inappropriate medication for their abdominal pain and cramping, including nonsteroidal anti-inflammatory drugs (NSAIDs), paracetamol and codeine-containing preparations. Overall, $85 \%$ of pharmacists recommended Buscopan for the treatment of abdominal pain and cramping, based on perceptions of efficacy, tolerability and improvement in quality of life.

Conclusions: Consumers don't always select an appropriate medication for abdominal pain and cramping, and require advice from a pharmacy HCP. Fostering trust and confidence is essential to ensure that the patient follows the advice given. Most South African pharmacy HCPs within these two major retail chain pharmacies recommend Buscopan as first line for treatment of abdominal cramps.

\section{Background}

Abdominal pain and cramping are extremely common. The prevalence in the general population has been estimated at between $10-46 \%$, with women reporting symptoms more commonly than men. ${ }^{1}$ These nonspecific symptoms may be associated with a wide spectrum of gastrointestinal (GI), urological and gynaecological disorders and, even in a hospital setting and despite use of imaging modalities, abdominal pain presents a major diagnostic challenge. ${ }^{2-4}$

Although most abdominal pain is benign and not lifethreatening, it can nevertheless have a substantial impact on quality of life.5,6 Functional GI disorders (FGID) are a common cause of painful abdominal cramps, and are characterised by Gl symptoms related to a combination of motility disturbance, visceral hypersensitivity, altered mucosal and immune function, altered gut microbiota, and altered central nervous system (CNS) processing. ${ }^{1,3}$ Up to $50 \%$ of these symptoms can be attributed to irritable bowel syndrome (IBS), an FGID symptom complex of abdominal pain and cramping, with other digestive symptoms including constipation, diarrhoea, bloating and flatulence. ${ }^{1}$ IBS significantly impacts work and social life. One study indicated that reduction in quality of life is severe enough that, on average, patients would sacrifice between 10 and 15 years of their remaining life expectancy for an immediate cure. ${ }^{6}$ The current standard treatment recommendations for abdominal pain and discomfort are to use a symptom-directed approach with medication aimed at alleviating pain, constipation and/or diarrhoea. Antimuscarinic agents are recommended first line for symptomatic management of abdominal cramps. ${ }^{2}$

A substantial proportion of people with Gl symptoms never consult a doctor and remain without a specific diagnosis. ${ }^{2}$ Multinational surveys have shown that the majority of people with intermittent cramping and pain use OTC medication, keep stock at home and rarely use medication for prevention, but rather on demand when pain occurs. ${ }^{1,7}$ Consequently, when asked what they expect from their medication, consumers consistently rate fast onset of action, long-lasting action, highly effective and good tolerability as most important. ${ }^{2,7}$ Antispasmodics are the most common class of therapeutic agents used by consumers. ${ }^{1,7}$

Buscopan (hyoscine butylbromide, HBB) is an anticholinergic antispasmodic medication indicated for the treatment of abdominal pain associated with cramps induced by Gl spasms. It has been available both on prescription and OTC for almost 70 years. $^{8}$ The muscle relaxing/antispasmodic effect of HBB is due to its high affinity for muscarinic receptors located on the smooth muscles of the Gl tract. It also binds to nicotinic receptors, inducing a ganglion-blocking effect. Numerous clinical trials have demonstrated its efficacy in abdominal pain associated with cramping. Because it is barely absorbed after oral administration and does not penetrate the blood-brain barrier it is generally well tolerated and few adverse events (in 
particular no significant increase in anticholinergic side-effects) have been reported. ${ }^{8}$

Currently there are no data evaluating the approach of South African pharmacy staff to abdominal cramps. Consequently, we conducted a survey to investigate the experience of pharmacy $\mathrm{HCPs}$ in two leading pharmacy retailers and their treatment recommendations for abdominal cramps, with a particular emphasis on attitudes towards Buscopan. The main objectives were to evaluate reasons for recommending Buscopan, to assess the level of knowledge about its efficacy, tolerability and perceived impact on quality of life, and to investigate other factors that might influence recommendations for management of abdominal pain and cramps.

\section{Methods}

The study recruited 142 pharmacists and 82 pharmacy assistants from two large retail pharmacy chains, who each completed a survey questionnaire either online $(n=74)$ or in a face-to-face interview $(n=150)$. Approximately $60 \%$ of respondents were female. The majority (>30\%) had more than four years' experience working in the pharmacy, whereas $47 \%$ of pharmacists and $28 \%$ of pharmacist's assistants had more than eight years' experience. As different pharmacies and retail groups have various recommendation algorithms and standard operating procedures (SOPs), these two chains were chosen to enable consistency in process and compliance programmes.

Results

Both pharmacists and pharmacist's assistants reported providing advice to an average of 13 people with abdominal cramps per week. Almost half of the pharmacists (47\%) and $44 \%$ of pharmacist's assistants reported seeing more than 20 patients with cramps per week. The most common causes of cramps (60$70 \%$ ) or associated symptoms were related to diarrhoea/upset tummy, IBS and menstruation-related cramps. Approximately half of the pharmacy HCPs reported providing advice to people with gastroenteritis or food poisoning, $40 \%$ to those with cramps attributed to gastrointestinal ulcers and $30 \%$ to those with cramps attributed to side-effects of antibiotics or other medications. With few exceptions, both pharmacists (96\%) and pharmacist's assistants (100\%) recommended a specific treatment to patients with abdominal cramps. Their primary considerations when recommending a treatment were cause of symptoms (pharmacists 69\%, pharmacist's assistants 61\%), potential for drug interactions $(61 \% ; 60 \%)$, price $(56 \% ; 51 \%)$ and safety profile (53\%; 45\%). Only approximately one in five pharmacy HCPs considered comorbidities as important when recommending treatment. Whereas $56 \%$ of pharmacists reported that patients followed their advice all of the time, fewer interactions with pharmacist's assistants led to patients always following the advice (41\%). The majority of pharmacy HCPs reported seeing a large number of patients ( $>6$ patients per week) who had treated their abdominal cramps with nonsteroidal anti-inflammatory drugs (NSAIDs). Approximately half said the average dose was 3-4 NSAID tablets per day, whereas one out of four had provided advice to patients taking 5-6 tablets or more per day. While menstruation-related cramps were a common reason for NSAID use, these medicines were also being used for cramps associated with IBS, diarrhoea, nausea/upset stomach and gastroenteritis. Almost half of the pharmacists had encountered patients who were taking NSAID medication for cramps attributed to stomach ulcers. Eighty-five per cent of pharmacy HCPs said they reminded all patients of the risks associated with NSAID use and maximum recommended doses. Similar observations were made for paracetamol and codeine-containing combination medications. However, only $75 \%$ of pharmacists and $67-70 \%$ of pharmacist's assistants reminded all of the patients about paracetamol- or codeine-associated risks and maximum doses.

More than $90 \%$ of these pharmacy HCPs were aware of Buscopan. Approximately $70-80 \%$ were aware of generic preparations

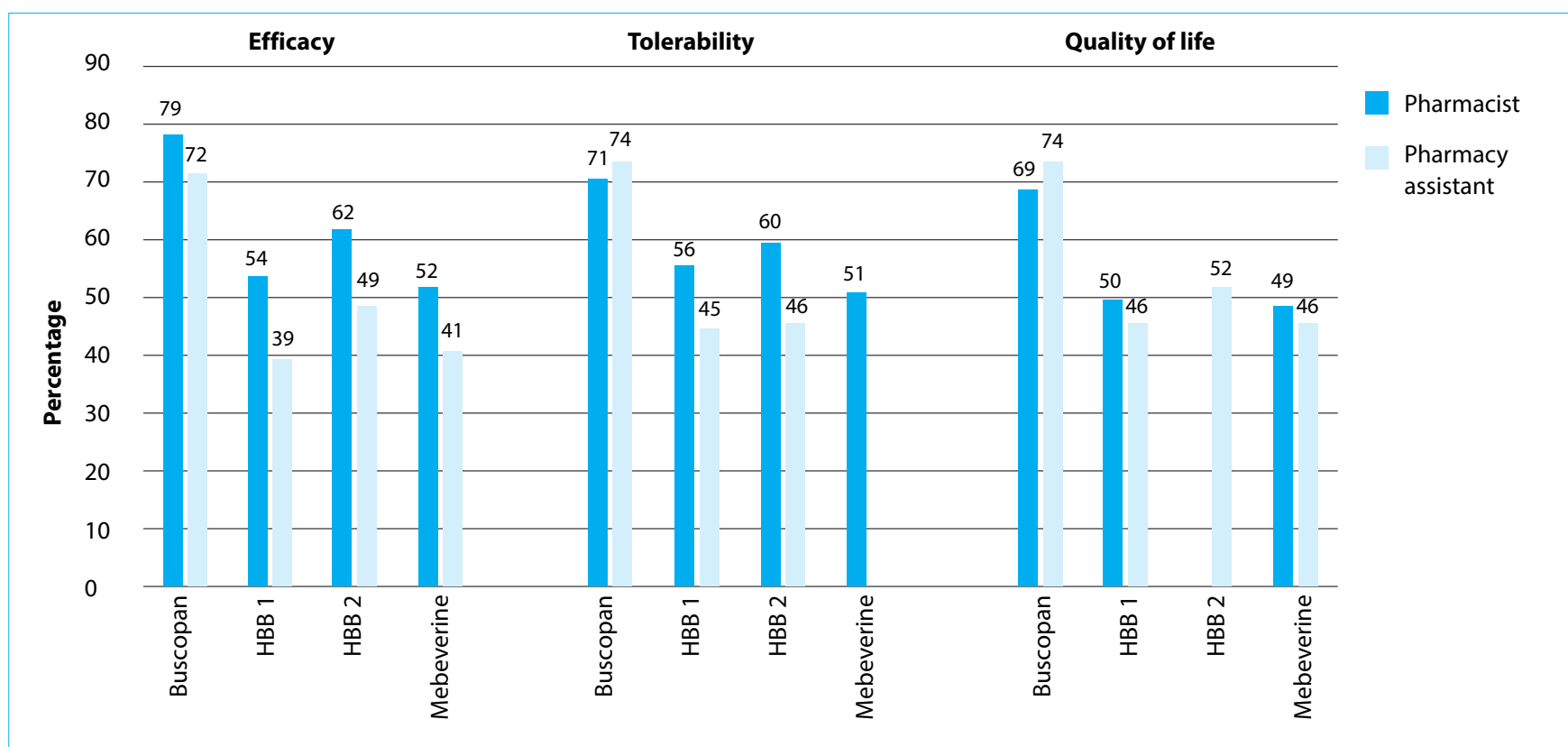

Figure 1: Recommendation practices for treatment of abdominal pain and cramps $\mathrm{HBB}$ - hyoscine butylbromide generic formulations 
of $\mathrm{HBB}$, mebeverine and a herbal preparation for treatment of abdominal cramps. Overall, $85 \%$ of pharmacists and $85 \%$ of pharmacist's assistants said that Buscopan was the most frequently recommended treatment for abdominal pain and cramps. The next most commonly recommended treatments were generic HBB (pharmacists $72 \%$; pharmacist's assistants $55 \%)$, mebeverine ( $66 \%$ and $46 \%$, respectively) and a herbal combination preparation ( $61 \%$ and $41 \%$, respectively). While all of the options were considered effective and tolerable for abdominal pain and cramps, when it came to recommendation practices, Buscopan was recommended most frequently, based on perceptions of efficacy, tolerability and improvement in quality of life (Figure 1). Pharmacist's assistants were also inclined to recommend a herbal product based on tolerability.

When asked what product attributes were most important to them when considering Buscopan, the majority of pharmacists (61\%) drew attention to the spasmolytic effect consequent to high affinity of HBB for muscarinic receptors on the smooth muscle cells of the Gl tract. More than half (56\%) rated good tolerability as an important consideration.

\section{Conclusions}

This survey demonstrates that, as in other parts of the world, South African pharmacy HCPs are commonly called upon to advise consumers about management of abdominal cramps. In line with treatment recommendations, as far as possible they take a symptom-based/diagnostic approach and the majority also consider the possibility of drug interactions before recommending treatment. However, comorbidities were less commonly considered. An alarming finding of the survey was that a substantial proportion of consumers do not always follow treatment recommendations and use of NSAIDs and other analgesic preparations appears to be common. While a NSAID might be an appropriate choice for patients with dysmenorrhoea, analgesics are not recommended as a general treatment for abdominal cramps. ${ }^{2}$ In particular the elderly and those with a history of peptic ulcer disease are at high risk of NSAID-associated complications and bleeding. ${ }^{9}$ Pharmacy HCPs did not always warn consumers of the risks associated with these medications.

Based on efficacy, tolerability and its perceived effect on quality of life, Buscopan was a leading recommended treatment for abdominal cramps in two major South African pharmacy retail chains. This is consistent with international guidelines that recommend an antispasmodic treatment as first-line therapy. ${ }^{2}$

In conclusion, it is common for patients to seek OTC treatment for abdominal cramps without a formal diagnosis. Consumers do not always select an appropriate medication and they require advice from a pharmacy $\mathrm{HCP}$ who can assist them with careful questioning about symptoms, other medications and comorbidities. Fostering trust and confidence is essential to ensure that the patient follows the advice given to them.

\section{Funding and conflicts of interest}

The survey was commissioned by Sanofi and conducted by Ipsos, an independent global market research agency which subscribes to the professional market research codes of conduct as set out by SAMRA and ESOMAR. Study design, collection, analysis and interpretation of data were independent of the sponsor. Ms Lara Jagwanth is the Medical Head for Africa for Sanofi CHC. Dr David Webb is a professional medical writer and was paid by Sanofi to write the report.

\section{References}

1. Quigley EMM, Locke GR, Mueller-Lissner S, et al. Prevalence and management of abdominal cramping and pain: a multinational survey. Aliment Pharmacol Ther. 2006;24:411-419. https://doi.org/10.1111/j.1365-2036.2006.02989.x.

2. World Gastroenterology Organisation. Coping with common gastrointestinal symptoms in the community. A global perspective on heartburn, constipation, bloating, and abdominal pain/discomfort May 2013. J Clin Gastroenterol. 2014;48(7):567-578. https://doi.org/10.1097/MCG.0000000000000141

3. Drossman DA. Functional gastrointestinal disorders: history, pathophysiology, clinical features, and Rome IV. Gastroenterology. 2016;150:1262-1279. https:// doi.org/10.1053/j.gastro.2016.02.032

4. Gans SL, Pols MA, Stoker J, Boermeeter MA on behalf of the expert steering group. Guideline for the diagnostic pathway in patients with acute abdominal pain. Dig Surg. 2015;32:23-31. https://doi.org/10.1159/000371583.

5. Cartwright SL, Knudson MP. Evaluation of abdominal pain in adults. Am Fam Phys. 2008;77(7):971-978.

6. Canavan C, West J, Card T. Review article: the economic impact of the irritable bowel syndrome. Aliment Pharmacol Ther. 2014;40:1023-1034. https://doi. org/10.1111/apt.12938.

7. Mueller-Lissner S, Quigley E, Helfrich I, Schaefer E. Drug treatment of chronic intermittent abdominal cramping and pain: a multi-national survey on usage and attitudes. Aliment Pharmacol Ther. 2010;32:472-477. https://doi. org/10.1111/j.1365-2036.2010.04368.x.

8. Tygat GN. Hyoscine butylbromide. A review of its use in the treatment of abdominal cramping and pain. Drugs. 2007;67(9):1343-1357. https://doi. org/10.2165/00003495-200767090-00007.

9. Lanza Fl, Chan FKL and the Practice Parameters Committee of the American College of Gastroenterology Guidelines for prevention of NSAID-related ulcer complications. Am J Gastroenterol. 2009;104:728-738. https://doi.org/10.1038/ ajg.2009.115. 


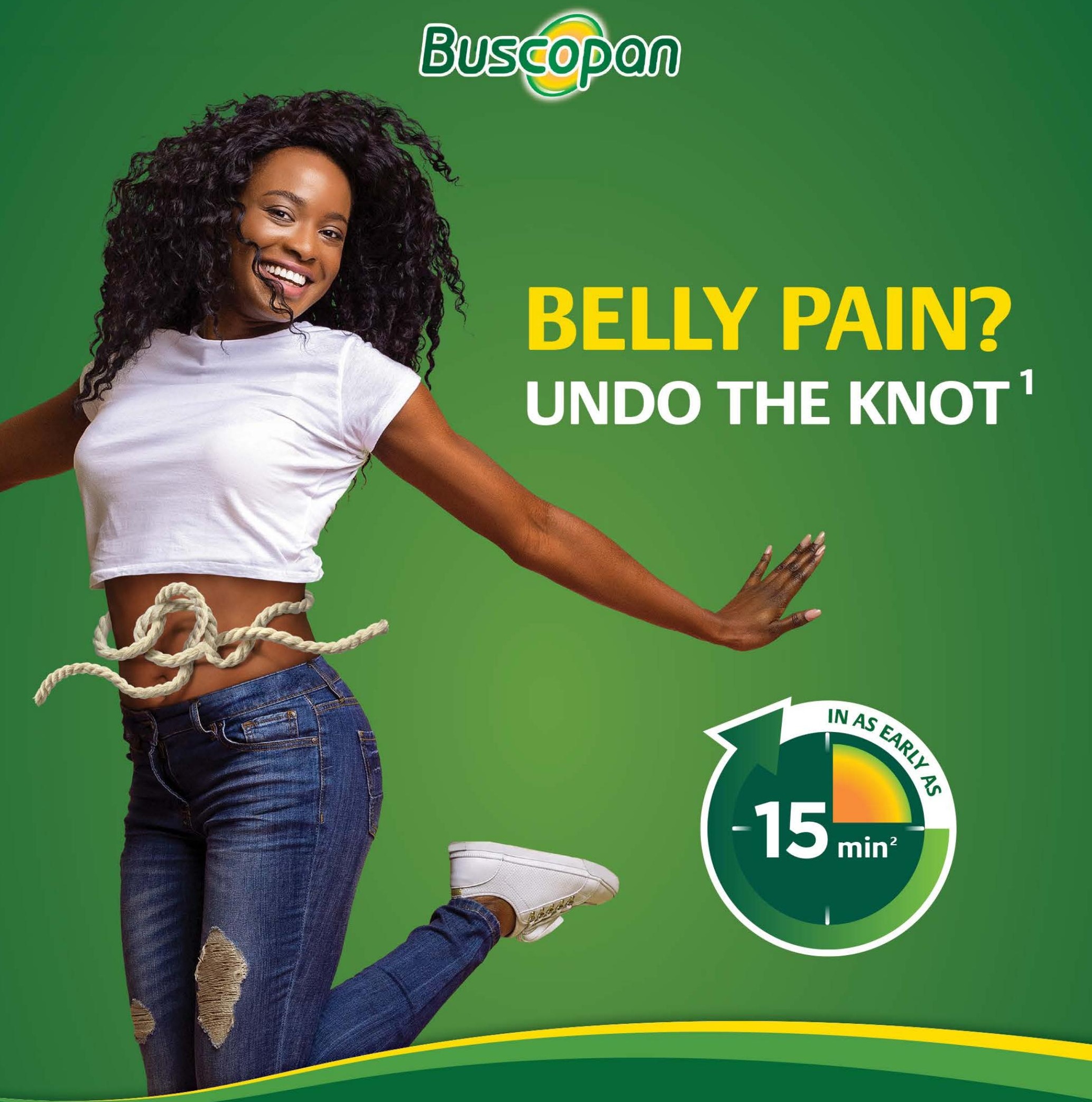

\section{UNDO THE KNOT. EMBRACE YOUR LIFE}

References: 1. BUSCOPAN 10 mg tablets package insert, 13 April 2003. 2. Lacy BE, Wang F. Bhowal S, Schaefer E; study group. On-demand hyoscine butylbromide for the treatment of self-reported functional cramping abdominal pain. Scand J Gastroenterol 2013 Aug;48(8):926-935.

S1 BUSCOPAN 10 mg tablets. Each tablet contains hyoscine butylbromide 10 mg. Reg. No. E 501 (Act 101/1965).

For full prescribing information refer to the package insert approved by the Medicines Regulatory Authority.

Applicant: sanofi-aventis south africa (pty) Itd, Reg. no.: 1996/010381/07. Sanofi House, 2 Bond Street, Grand Central Ext. 1, Midrand, 1685. Tel: (011) 2563700 Fax: (011) 256 3707. www.sanofi-aventis.com MAT-ZA-2000165-2.0-07/2020 